\title{
Influence of plasma environment on K-line emission in highly ionized iron atoms evaluated using a Debye-Hückel model ${ }^{1}$
}

\author{
J. Deprince, S. Fritzsche, T.R. Kallman, P. Palmeri, and P. Quinet
}

\begin{abstract}
The influence of plasma environment on the atomic parameters associated with the K-vacancy states has been investigated theoretically for several iron ions. To do this, a time-averaged Debye-Hückel potential for both the electron-nucleus and electron-electron interactions has been considered in the framework of relativistic multiconfiguration Dirac-Fock computations. More particularly, the plasma screening effects on ionization potentials, K-thresholds, transition energies, and radiative rates have been estimated in the astrophysical context of accretion disks around black holes. In the present paper, we describe the behaviour of those atomic parameters for Ne-, Na-, Ar-, and K-like iron ions.

Key words: ab initio calculations, properties of atoms, atomic spectra, X-ray spectra, inner-shell excitation and ionization.

Résumé : L'influence de l'environnement plasma sur les paramètres atomiques associés aux états comportant un trou dans la couche K a été étudiée théoriquement pour plusieurs ions du fer. Pour ce faire, un potentiel de Debye-Hückel écrantant les interactions electron-noyau et électron-électron a été considéré dans le cadre de la méthode relativiste multiconfigurationnelle de Dirac-Fock. Plus particulièrement, les effets du plasma sur les potentiels d'ionisation, les seuils K, les énergies de transition et les taux radiatifs ont été estimés pour des conditions astrophysiques typiques rencontrées dans les disques d'accrétion autour de trous noirs. Dans le présent article, nous décrivons le comportement de ces différents paramètres pour les ions du fer appartenant aux séquences isoélectroniques du néon, du sodium, de l’argon et du potassium.
\end{abstract}

Mots-clés : calculs ab initio, propriétés atomiques, spectres atomiques, spectres $\mathrm{X}$, excitation et ionisation des couches internes.

\section{Introduction}

The K-lines from iron ions are of paramount importance for investigating the physical conditions of astrophysical plasmas, such as those observed in accretion disks around black holes. These lines, appearing in the X-ray spectral region at about $6.4 \mathrm{keV}$, have observed widths and shifts that imply an origin very close to the compact object in many cases [1]. To interpret and model the $\mathrm{X}$-ray spectra recorded for such celestial objects, it is essential to know, with a high level of accuracy, the atomic parameters associated with K-lines for all the ionization stages of iron. If the determination of many of these parameters (transition energies, radiative transition probabilities, Auger rates, fluorescence yields, K-thresholds, photoabsorption and photoionization cross sections, and radiative recombination rates) has been the subject of different studies in the past [2-8], none of them took the plasma environment effects into account even though temperatures ranging from $10^{5}$ to $10^{7} \mathrm{~K}$ and electronic densities ranging from $10^{18}$ to $10^{21} \mathrm{~cm}^{-3}$ can be expected in the case of accretion disks around black holes, as predicted by recent magnetohydrodynamics (MHD) simulations [9]. It is worth noting that such high densities are required to allow the survival of iron ions against ionization near a black hole. Effects of plasma density can also be important in other environments, such as neutron stars, accreting white dwarf atmospheres, and partially ionized outflows from galactic sources [10]. The densities in these sources approach or exceed the limits of applicability for many of the rates in current atomic databases, thus new atomic calculations tailored for high-density plasmas are greatly needed.

In our work, we estimated the influence of plasma environment on the atomic parameters associated with the K-vacancy states in iron ions. The theoretical method used is based on the purely relativistic multiconfiguration Dirac-Fock (MCDF) approach in which the plasma screening effects are considered by means of a time-averaged Debye-Hückel potential for both the electronnucleus and electron-electron electrostatic interactions. The behaviour of different atomic parameters, such as the ionization potentials, the K-thresholds, the transition energies, and the radiative emission rates for some iron ions characterized by rather simple ground configurations with closed shells or only one valence electron (i.e., Ne-like Fe XVII, Na-like Fe XVI, Ar-like Fe IX, and K-like Fe VIII) is discussed in the present paper. This represents an extension of similar results recently published in the case of He-like Fe XXV and Li-like Fe XXIV [11].

\section{Theoretical calculations}

To describe the effects of the plasma screening on the atomic properties of multiply charged iron ions, we considered a DebyeHückel potential, which can be expressed, in atomic units (a.u.), by

Received 13 September 2016. Accepted 13 March 2017.

J. Deprince and P. Palmeri. Physique Atomique et Astrophysique, Université de Mons, B-7000 Mons, Belgium.

S. Fritzsche. Helmholtz Institut Jena, 07743 Jena, Germany; Theoretisch Physikalisches Institut, Friedrich Schiller Universität Jena, 07743 Jena, Germany.

T.R. Kallman. NASA Goddard Space Flight Center, Code 662, Greenbelt, MD, USA.

P. Quinet. Physique Atomique et Astrophysique, Université de Mons, B-7000 Mons, Belgium; IPNAS, Université de Liège, B-4000 Liège, Belgium.

Corresponding author: P. Quinet (email: Pascal.Quinet@umons.ac.be).

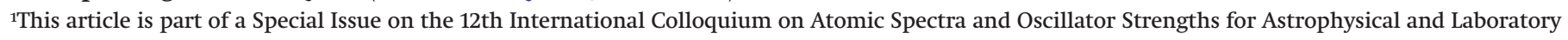
Plasmas.

Copyright remains with the author(s) or their institution(s). Permission for reuse (free in most cases) can be obtained from RightsLink. 


\section{Pagination not final (cite DOI) / Pagination provisoire (citer le DOI)}

Table 1. Values of the screening parameter, $\lambda$ in a.u. for different plasma conditions.

\begin{tabular}{lllll}
\hline & $\lambda$ & & \\
\cline { 2 - 5 } $\mathrm{T}_{\mathrm{e}}(\mathrm{K})$ & $n_{\mathrm{e}}=10^{18} \mathrm{~cm}^{-3}$ & $n_{\mathrm{e}}=10^{19} \mathrm{~cm}^{-3}$ & $n_{\mathrm{e}}=10^{20} \mathrm{~cm}^{-3}$ & $n_{\mathrm{e}}=10^{21} \mathrm{~cm}^{-3}$ \\
\hline $10^{5}$ & 0.002 & 0.008 & 0.024 & 0.077 \\
$10^{6}$ & 0.001 & 0.002 & 0.008 & 0.024 \\
$10^{7}$ & 0.000 & 0.001 & 0.002 & 0.008 \\
\hline
\end{tabular}

$V^{\mathrm{DH}}(r, \lambda)=-\sum_{i=1}^{N} \frac{Z \mathrm{e}^{-\lambda r_{i}}}{r_{i}}+\sum_{i>j}^{N} \frac{\mathrm{e}^{-\lambda r_{i j}}}{r_{i j}}$

where $N$ is the number of bound electrons, $r_{i}$ is the distance of the $i$ th electron from the nucleus, and $r_{i j}$ is the distance between the electrons $i$ and $j$. The plasma screening parameter, $\lambda$, is the inverse of the Debye shielding length, $\lambda_{\text {De }}$, and can be written in terms of the electron density, $n_{\mathrm{e}}$, and temperature, $T_{\mathrm{e}}$, of the plasma as

$\lambda=\frac{1}{\lambda_{\text {De }}}=\left(\frac{4 \pi n_{\mathrm{e}}}{k \mathrm{~T}_{\mathrm{e}}}\right)^{1 / 2} \quad$ (a.u.)

Any given $\lambda$ is associated with a certain plasma environment. In the atomic structure calculations herein, we shall then replace the electron-nucleus and electron-electron interactions of the ion by the corresponding Debye-Hückel potential given in (1) and modify the screening by means of the parameter $\lambda$. In the astrophysical context of an accretion disk around a black hole, MHD simulations [9] performed for accreting black holes with 10 solar masses and an accretion rate of $10 \%$ established that the plasma parameters should be $T_{\mathrm{e}}=10^{5}-10^{7} \mathrm{~K}$ and $n_{\mathrm{e}}=10^{18}-10^{21} \mathrm{~cm}^{-3}$. This corresponds to values up to about 0.1 a.u. for the screening parameter, as shown in Table 1.

All the atomic structure and radiative rate calculations were carried out with the purely relativistic MCDF method [12-14] implemented in the GRASP92 [15] and RATIP [16] computer packages. In this method, the atomic state functions, $\Psi\left(\gamma \mathrm{J} M_{\mathrm{J}}\right)$, are expanded in linear combinations of configuration state functions (CSFs), $\Phi\left(\alpha_{i} J M_{\mathrm{J}}\right)$, according to

$\Psi\left(\gamma J M_{J}\right)=\sum_{i} c_{i} \Phi\left(\alpha_{i} J M_{J}\right)$

The CSFs are taken as antisymetrized products of a common set of orthonormal monoelectronic spin-orbitals of the form

$\varphi_{n \kappa m}(r, \theta, \phi)=\frac{1}{r}\left(\begin{array}{c}P_{n \kappa}(r) \chi_{\kappa m}(\theta, \phi) \\ i Q_{n \kappa}(r) \chi_{-\kappa m}(\theta, \phi)\end{array}\right)$

where $P_{n \kappa}(r)$ and $Q_{n \kappa}(r)$ are the large and the small components, respectively, of the radial wavefunctions. The $\alpha_{i}$ represent all the one-electron and intermediate quantum numbers needed to completely define the CSF while $\gamma$ is usually chosen as the $\alpha_{i}$ corresponding to the CSF with the largest weight $\left|c_{i}\right|^{2}$. The quantum number $\kappa$ is given by

$\kappa= \pm\left(j+\frac{1}{2}\right)$

where $j$ is the electron total angular momentum. The sign before the parenthesis in (5) corresponds to the coupling relation between the electron orbital momentum, $\ell$, and its spin, that is, $\ell=j \pm \frac{1}{2}$

The angular functions $\chi_{\kappa m}(\theta, \phi)$ are the spinor spherical harmonics in the $\ell s j$ coupling scheme. Their expression is given, for example, by [17]:

$\chi_{\kappa m}(\theta, \phi)=\sum_{\sigma= \pm 1 / 2}\left\langle\ell, m-\sigma, \frac{1}{2}, \sigma \mid \ell, \frac{1}{2}, j, m\right\rangle Y_{l}^{m-\sigma}(\theta, \phi) \xi_{\sigma}$

where $\xi_{\sigma}$ are the two-spinors

$\xi_{1 / 2}=\left(\begin{array}{l}1 \\ 0\end{array}\right) \quad \xi_{-1 / 2}=\left(\begin{array}{l}0 \\ 1\end{array}\right)$

The radial functions $P_{n \kappa}(r)$ and $Q_{n \kappa}(r)$ are numerically represented on a logarithmic grid and are required to be orthonormal within each $\kappa$ symmetry. In the MCDF variational procedure, the radial functions and the expansion coefficients $c_{i}$ are optimized to self-consistency.

As pointed out in different previous papers (see, e.g., refs. 18-21), there remains some controversy about the choice of screening potential to model the plasma effect on the atomic structures and radiative processes. As a reminder, the plasma environment can be classified into weakly and strongly coupled plasma depending on the strength of its coupling parameter, $\Gamma$, which is the ratio of the Coulomb potential interaction energy between two particles to the thermal energy, and which can be expressed by [22]

$\Gamma=\frac{e^{2}}{4 \pi \varepsilon_{0} d k T_{\mathrm{e}}}$

for particles separated by a typical distance $d$, such as

$d=\left(\frac{3}{4 \pi n_{\mathrm{e}}}\right)^{1 / 3}$

For weakly coupled plasmas $(\Gamma \ll 1)$, it is well known that the screening effect can be appropriately described using the DebyeHückel model while, for strongly coupled plasmas $(\Gamma \gg 1)$, the ion-sphere potential model is the best suited model to account for the plasma screening effects (see, e.g., ref. 21). In the conditions of electronic densities $\left(10^{18}-10^{21} \mathrm{~cm}^{-3}\right)$ and temperatures $\left(10^{5}-10^{7} \mathrm{~K}\right)$ investigated in our work (see Table 1), we can easily note that the coupling parameter $\Gamma$ is always lower (much lower in most cases) than unity, that is, the statically shielded Debye-Hückel potential should be appropriate to describe the screened Coulomb interaction. Moreover, the importance of screening effects on electronelectron interaction was highlighted very recently by Das et al. [23] who found a significant influence of such effects on the ionization potential depressions and excitation energies in $\mathrm{Al}$ ions, the neutral and lowly ionized species being more affected than the highly ionized ones, the former having more electrons than the latter. In the same paper, it was also shown that, in similar plasma conditions (i.e., in the intermediate region between weakly and strongly coupled plasmas, around $\Gamma \approx 1$ ) both Debye-Hückel and ion-sphere models reasonably agree with each other. For these reasons, the DebyeHückel potential given in (1) was used in the present work.

Nevertheless, to test our method, we computed the redshift of the Ti He- $\alpha$ line in the same conditions as those considered in the ion-sphere approach used by Belkhiri et al. [21], that is, an electron temperature of $3000 \mathrm{eV}\left(3.5 \times 10^{7} \mathrm{~K}\right)$ and an electron density of 
$4.2 \times 10^{24} \mathrm{~cm}^{-3}$. In this case, we found a line-shift of $3.3 \mathrm{eV}$, in very good agreement with their theoretical result (i.e., $3.4 \mathrm{eV}$ ) and with the experimental measurement reported by Khattak et al. [24]. Another example concerns the Ne X Lyman- $\alpha$ line for which our model gives a redshift of $24.5 \mathrm{eV}$ in the case of an electron density of $6 \times 10^{24} \mathrm{~cm}^{-3}$ and a temperature of $500 \mathrm{eV}\left(5.8 \times 10^{6} \mathrm{~K}\right)$. This line-shift value is in very good agreement with the results $(\sim 25-$ $26 \mathrm{eV}$ ) presented in fig. 2 of the paper published by Nguyen et al. [18] and obtained using different models, namely, those based on the quantum mechanical impact theory including the boundary depression effect, on the quantum mechanical impact theory using the volume averaged electron density, and on the confined atom in a self-consistent field.

In the present work, the atomic structures of K-like (Fe VIII), Ar-like (Fe IX), Na-like (Fe XVI), and Ne-like (Fe XVII) iron ions were investigated by using the active space method for building the MCDF multiconfiguration expansions. The latter are produced by exciting the electrons from the reference configurations to a given active set of orbitals. More precisely, for Fe VIII, the active set contained all the single excitations from the $1 s^{2} 2 s^{2} 2 p^{6} 3 s^{2} 3 p^{6} 3 d$ and $1 s^{2} 2 s^{2} 2 p^{6} 3 s^{2} 3 p^{6} 4 s$ configurations to the $3 d$ and $4 s$ orbitals. This gave rise to the following set of configurations: $1 \mathrm{~s}^{2} 2 \mathrm{~s}^{2} 2 \mathrm{p}^{6} 3 \mathrm{~s}^{2} 3 \mathrm{p}^{6} 3 \mathrm{~d}$, $1 s^{2} 2 s^{2} 2 p^{6} 3 s^{2} 3 p^{6} 4 s, 1 s 2 s^{2} 2 p^{6} 3 s^{2} 3 p^{6}\left(3 d^{2}, 3 d 4 s, 4 s^{2}\right), 1 s^{2} 2 s^{2} p^{6} 3 s^{2} 3 p^{6}$ $\left(3 d^{2}, 3 d 4 s, 4 s^{2}\right), 1 s^{2} 2 s^{2} 2 p^{5} 3 s^{2} 3 p^{6}\left(3 d^{2}, 3 d 4 s, 4 s^{2}\right), 1 s^{2} 2 s^{2} 2 p^{6} 3 s 3 p^{6}\left(3 d^{2}\right.$, $\left.3 d 4 s, 4 s^{2}\right)$, and $1 s^{2} 2 s^{2} 2 p^{6} 3 s^{2} 3 p^{5}\left(3 d^{2}, 3 d 4 s, 4 s^{2}\right)$. For Fe IX, all the single excitations from the $1 s^{2} 2 s^{2} 2 p^{6} 3 s^{2} 3 p^{6}$ ground configuration to the $3 \mathrm{~d}$ and $4 \mathrm{~s}$ orbitals were considered in the MCDF calculations. This led to the following multiconfiguration expansion: $1 s^{2} 2 s^{2} 2 p^{6} 3 s^{2} 3 p^{6}, 1 s 2 s^{2} 2 p^{6} 3 s^{2} 3 p^{6}(3 d, 4 s), 1 s^{2} 2 s 2 p^{6} 3 s^{2} 3 p^{6}(3 d, 4 s)$, $1 s^{2} 2 s^{2} 2 p^{5} 3 s^{2} 3 p^{6}(3 d, 4 s), 1 s^{2} 2 s^{2} 2 p^{6} 3 s^{3} p^{6}(3 d, 4 s)$, and $1 s^{2} 2 s^{2} 2 p^{6} 3 s^{2} 3 p^{5}$ (3d, $4 \mathrm{~s})$. In the case of Fe XVI, the set of configurations used in the model was $1 s^{2} 2 s^{2} 2 p^{6}(3 s, 3 p), 1 s^{2} 2 s^{2} 2 p^{5}\left(3 s^{2}, 3 s 3 p, 3 p^{2}\right), 1 s^{2} 2 s 2 p^{6}\left(3 s^{2}\right.$, $\left.3 s 3 p, 3 p^{2}\right)$, and $1 s 2 s^{2} 2 p^{6}\left(3 s^{2}, 3 s 3 p, 3 p^{2}\right)$. These were generated by all the single excitations from $1 s^{2} 2 s^{2} 2 p^{6} 3 s$ and $1 s^{2} 2 s^{2} 2 p^{6} 3 p$ to the 3 s and $3 p$ orbitals. Finally, for Fe XVII, all the single excitations from the $1 s^{2} 2 s^{2} 2 p^{6}$ ground configuration to the $3 s$ and $3 p$ orbitals were considered, which corresponds to the following multiconfiguration expansion: $1 s^{2} 2 s^{2} 2 p^{6}, 1 s^{2} 2 s^{2} 2 p^{5}(3 s, 3 p), 1 s^{2} 2 s 2 p^{6}(3 s, 3 p)$, and $1 \mathrm{~s} 2 \mathrm{~s}^{2} 2 \mathrm{p}^{6}(3 \mathrm{~s}, 3 \mathrm{p})$. The computations were done with the extended average level option, optimizing a weighted trace of the Hamiltonian using level weights proportional to $2 J+1$, and they were completed with the inclusion of the relativistic two-body Breit interaction and the quantum electrodynamic corrections due to self-energy and vacuum polarization. The MCDF ionic bound states generated by the GRASP92 code were then used in the RATIP program [16] to compute the atomic structure and radiative parameters associated with the K-vacancy states.

\section{Results and discussion}

\subsection{Ionization potentials and K-thresholds}

By including the Debye-Hückel potential given in (1) into the MCDF equations, we estimated the influence of different plasma conditions on the atomic properties using different values of the screening parameter, $\lambda$, taken from Table 1 . The results obtained for the ionization potentials and K-thresholds are summarized in Table 2 . We note that, when going from $\lambda=0$ (isolated ion) to $\lambda=$ 0.1 (which corresponds to typical plasma conditions, such as $T_{e}=$ $10^{5} \mathrm{~K}$ and $n_{\mathrm{e}}=10^{21} \mathrm{~cm}^{-3}$ ), the ionization potentials of Fe VIII, Fe IX, Fe XVI, and Fe XVII are reduced by 21.29, 24.29, 42.17, and $45.85 \mathrm{eV}$, while the corresponding K-thresholds are reduced by 22.54, 24.86, 43.61 , and $46.20 \mathrm{eV}$, respectively. It is worth mentioning that, although this ionization potential depression in dense plasmas is a well-known process, this is not taken into account in a comprehensive way in current ionization balance calculations carried out in photoionized plasma modeling. As an example, the code XSTAR $[25,26]$, which is widely used for modeling synthetic photoionized $\mathrm{X}$-ray spectra, and which incorporates the most complete and
Table 2. Computed ionization potentials and K-thresholds for Fe VIII, Fe IX, Fe XVI, and Fe XVII ions as a function of the plasma screening parameter $\lambda$, in a.u.

\begin{tabular}{lrrlllll}
\hline & \multicolumn{3}{l}{ Ionization potential $(\mathrm{eV})$} & & \multicolumn{3}{l}{ K-threshold $(\mathrm{eV})$} \\
\cline { 2 - 3 } Ion & $\lambda=0.0$ & $\lambda=0.1$ & Shift & & $\lambda=0.0$ & $\lambda=0.1$ & Shift \\
\hline Fe VIII & 150.73 & 129.44 & -21.29 & & 7304.78 & 7282.24 & -22.54 \\
Fe IX & 241.24 & 217.59 & -24.25 & & 7333.58 & 7308.72 & -24.86 \\
Fe XVI & 489.24 & 447.07 & -42.17 & & 7659.83 & 7616.22 & -43.61 \\
Fe XVII & 1263.73 & 1217.88 & -45.85 & & 7707.95 & 7661.75 & -46.20 \\
\hline
\end{tabular}

Table 3. Comparison between the transition energies $(\Delta E)$ and the radiative transition probabilities $\left(A_{k i}\right)$ computed for the strongest $\mathrm{K}_{\alpha}$-lines $\left(A_{k i}>10^{14} \mathrm{~s}^{-1}\right)$ in Fe VIII, Fe IX, Fe XVI, and Fe XVII using a plasma screening parameter $\lambda=0.1$ and the values obtained for an isolated ion $(\lambda=0.0)$.

\begin{tabular}{|c|c|c|c|c|c|}
\hline \multirow[b]{2}{*}{ Transition } & \multicolumn{2}{|l|}{$\Delta E(\mathrm{eV})$} & \multirow{2}{*}{$\begin{array}{l}\text { Shift } \\
(\mathrm{eV})\end{array}$} & \multicolumn{2}{|c|}{$A_{k i}\left(10^{14} \mathrm{~s}^{-1}\right)$} \\
\hline & $=0.0$ & $\lambda=0.1$ & & $=0.0$ & $\lambda=0$. \\
\hline \multicolumn{6}{|l|}{ Fe VIII } \\
\hline $\begin{array}{l}{[1 s] 3 d^{2}{ }^{4} F_{5}} \\
\text { Fe IX }\end{array}$ & 20.41 & 6420.01 & -0.40 & 2.059 & .059 \\
\hline$\left.d^{1} D_{-}-2 \mathrm{pl}\right] 3 \mathrm{~d}^{3} \mathrm{~F}_{2}$ & 06.98 & 6406.58 & -0.40 & 309 & 309 \\
\hline & 07.49 & 6407.09 & -0.40 & 60 & .260 \\
\hline$F_{2}$ & 407.51 & 6407.11 & -0.40 & .053 & 1.053 \\
\hline $\mathrm{O}_{3}$ & 418.46 & 6418.06 & -0.40 & 276 & 1.275 \\
\hline & & & & 87 & 1.488 \\
\hline 2 & & & & & 607 \\
\hline $2 \mathrm{p}] 3 \mathrm{~d}^{3} \mathrm{P}_{1}$ & & & & & .052 \\
\hline 4D & & & -0.42 & 01 & .994 \\
\hline - & 428.66 & 6428.24 & -0.42 & 341 & 341 \\
\hline & 428.82 & 6428.41 & -0.41 & & 250 \\
\hline$[21$ & 429.63 & 642 & 42 & & 920 \\
\hline$P_{1}$ & 430.94 & 643 & -0.44 & 84 & .081 \\
\hline$P_{5 / 2}$ & .76 & 34 & -0.42 & 29 & .624 \\
\hline 72 & 27 & & -0.46 & 25 & 624 \\
\hline 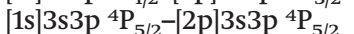 & & & & & 186 \\
\hline 2 & 6438.38 & & -0.42 & & 209 \\
\hline $2 \mathrm{D}^{3 / 2}$ & 6439.81 & 643 & -0.43 & 88 & 497 \\
\hline $5 / 2$ & 440.12 & & $-c$ & 54 & .763 \\
\hline & & & 13 & & 621 \\
\hline 2 & 2.10 & & 43 & & 728 \\
\hline$[2 \mathrm{p}$ & 6442.49 & 6442.06 & -0.43 & 3.914 & .914 \\
\hline $4 \mathrm{D}$ & 6442.53 & & -0.42 & 48 & .045 \\
\hline $2 D^{2}$ & & & & & .113 \\
\hline & & & - & & .031 \\
\hline$-[2 p] 3 s 3 p{ }^{4} D_{3 / 2}$ & 64 & 9 & - & $0_{0}$ & 年 \\
\hline & & & - & & .041 \\
\hline & & & & & 36 \\
\hline & 5 & 2 & $-c$ & 1.082 & .082 \\
\hline$P_{1}-[2 \mathrm{~F}$ & 6433.46 & 6433.04 & -0.42 & 1.522 & 1.522 \\
\hline$[1 \mathrm{~s}$ & 6436.25 & 6435.83 & -0.42 & 2.699 & 2.699 \\
\hline${ }^{3} \mathrm{P}_{0}-[2 \mathrm{p}] 3 \mathrm{p}^{1} \mathrm{P}_{1}$ & 6440.80 & & -0.42 & 1.817 & 1.819 \\
\hline & & & -0.43 & 3.011 & .010 \\
\hline & 6444.95 & & -0.43 & 2.779 & .778 \\
\hline${ }^{1} \mathrm{P}_{1}-[2]$ & & 64 & & & 1.478 \\
\hline${ }^{3} \mathrm{~S}_{1}-[2 \mathrm{p}]$ & 6445.43 & 6445.00 & -0.43 & 3.283 & 3.282 \\
\hline$[1 s] 3 p^{1} P_{1}-[2 p] 3 p^{11}$ & 6446.79 & 6446.36 & -0.43 & 1.111 & 1.110 \\
\hline & & & & 2.201 & 2.199 \\
\hline & 6448.47 & 6448.04 & -0.43 & 3.224 & 3.22 \\
\hline
\end{tabular}

detailed database for the K-shell atomic properties of many astrophysically relevant ions, is also limited to densities up to $10^{18} \mathrm{~cm}^{-3}$. In addition, other atomic processes, such as photoionization, Auger effect, radiative recombination, dielectronic recombination, and electron impact collisions, can be affected by the ionization potential lowering. 
Fig. 1. Variation of the transition energies, $\Delta E$, with the plasma screening parameter $\lambda$ for two K-lines in Fe IX.

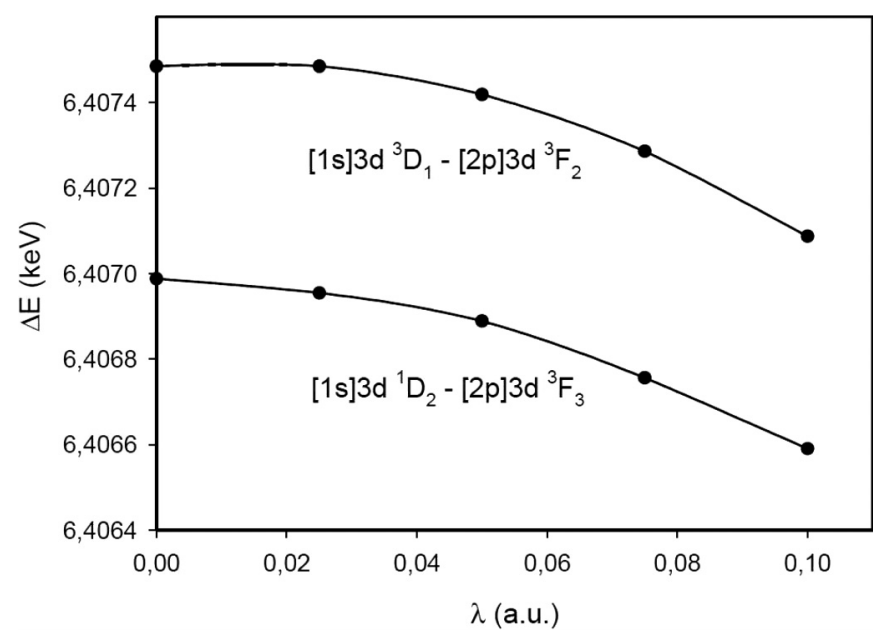

Fig. 2. Variation of the transition probabilities, $A_{k i}$, with the plasma screening parameter $\lambda$ for two K-lines in Fe XVI.

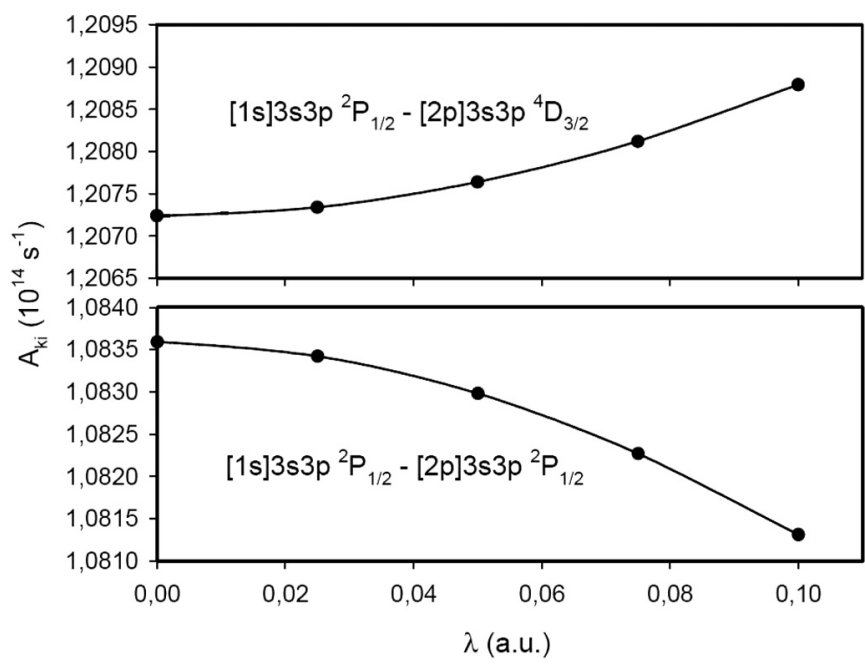

\subsection{Transition energies and radiative rates}

The transition energies, $\Delta E$ in electronvolts, and radiative transition probabilities, $A_{k i}$ in inverse seconds, computed in our work for the strongest $\left(A_{k i}>10^{14} \mathrm{~s}^{-1}\right) \mathrm{K}_{\alpha}$ lines in Fe VIII, Fe IX, Fe XVI, and Fe XVII are reported in Table 3 . These results were obtained using two different values of the plasma screening parameter (i.e., $\lambda=0.0$ and 0.1 a.u.). When looking at the table, we can see that the plasma environment reduces all transition energies by about $0.4-0.5 \mathrm{eV}$. This is illustrated in Fig. 1 showing the behaviour of $\Delta E$ for two particular transitions in Ar-like Fe IX (i.e., $1 \mathrm{~s} 2 \mathrm{~s}^{2} 2 \mathrm{p}^{6} 3 \mathrm{~s}^{2} 3 \mathrm{p}^{6} 3 \mathrm{~d}{ }^{3} \mathrm{D}_{1}-$ $1 s^{2} 2 s^{2} 2 p^{5} 3 s^{2} 3 p^{6} 3 d^{3} F_{2}$ and $1 s 2 s^{2} 2 p^{6} 3 s^{2} 3 p^{6} 3 d^{1} D_{2}-1 s^{2} 2 s^{2} 2 p^{5} 3 s^{2} 3 p^{6} 3 d$ ${ }^{3} \mathrm{~F}_{3}$ ) with respect to the plasma conditions. From Table 3, we can note that the changes observed for the radiative transition rates do not exceed $0.4 \%$. Although rather small, it is however interesting to mention that these changes do not exhibit any systematic trend; some $A$-values increase while others decrease when going from $\lambda=0.0$ to 0.1 a.u. As an example, Fig. 2 shows the behaviour of the calculated transition probabilities corresponding to the $1 \mathrm{~s} 2 \mathrm{~s}^{2} 2 \mathrm{p}^{6} 3 \mathrm{~s} 3 \mathrm{p}{ }^{2} \mathrm{P}_{1 / 2}-$ $1 s^{2} 2 s^{2} 2 p^{5} 3 s 3 p{ }^{4} D_{3 / 2}$ and $1 s 2 s^{2} 2 p^{6} 3 s 3 p{ }^{2} P_{1 / 2}-1 s^{2} 2 s^{2} 2 p^{5} 3 s 3 p{ }^{2} P_{1 / 2} K_{\alpha}$ lines in Na-like Fe XVI.

\section{Conclusion}

The effects of plasma environment on the atomic structure and radiative emission rates associated with $\mathrm{K}$-lines in $\mathrm{Ne}-$, $\mathrm{Na}-, \mathrm{Ar}-$, and K-like iron ions have been estimated using a Debye-Hückel model included within the relativistic MCDF approach. It has been shown that, for typical astrophysical conditions met in accretion disks around black holes of 10 solar masses $\left(T_{e}=10^{5}-10^{7} \mathrm{~K}\right.$, $n_{\mathrm{e}}=10^{18}-10^{21} \mathrm{~cm}^{-3}$ ), the ionization potentials as well as the K-thresholds can be reduced by values ranging from about $22 \mathrm{eV}$ (for Fe VIII) up to about $46 \mathrm{eV}$ (for Fe XVII). On the other hand, we found that the K-inner shell transition energies are reduced by 0.4-0.5 eV, while the corresponding radiative transition rates are slightly affected by at most $0.4 \%$. In the near future, we intend to apply the same method as reported in the present paper to study more complex iron ions, from Fe I to Fe XXIII, for which we can expect that the electron-electron interaction is more affected by the screening effects. We also plan to extend the investigation of the influence of plasma environment on other atomic processes, such as Auger effect, radiative recombination, photoabsorption, and photoionization in all iron ions.

\section{Acknowledgements}

J.D. is the holder of a FRIA Fellowship. P.P. and P.Q. are Research Associate and Research Director, respectively, of the Belgian Fund for Scientific Research F.R.S.-FNRS. Financial support from this organization is gratefully acknowledged.

\section{References}

1. C.S. Reynolds and M.A. Nowak. Phys. Rep. 377, 389 (2003). doi:10.1016/S03701573(02)00584-7.

2. P. Palmeri, C. Mendoza, T.R. Kallman, and M.A. Bautista. Astrophys. J. 577, L119 (2002). doi:10.1086/344243.

3. M.A. Bautista, C. Mendoza, T.R. Kallman, and P. Palmeri. Astron. Astrophys. 403, 339 (2003). doi:10.1051/0004-6361:20030367.

4. P. Palmeri, C. Mendoza, T.R. Kallman, and M.A. Bautista. Astron. Astrophys. 403, 1175 (2003). doi:10.1051/0004-6361:20030405.

5. P. Palmeri, C. Mendoza, T.R. Kallman, M.A. Bautista, and M. Meléndez. Astron. Astrophys. 410, 359 (2003). doi:10.1051/0004-6361:20031262.

6. C. Mendoza, T.R. Kallman, M.A. Bautista, and P. Palmeri. Astron. Astrophys. 414, 377 (2004). doi:10.1051/0004-6361:20031621.

7. T.R. Kallman, P. Palmeri, M.A. Bautista, C. Mendoza, and J.H. Krolik. Astrophys. J. Suppl. Ser. 155, 675 (2004). doi:10.1086/424039.

8. M.A. Bautista, C. Mendoza, T.R. Kallman, and P. Palmeri. Astron. Astrophys. 418, 1171 (2004). doi:10.1051/0004-6361:20034198.

9. J.D. Schnittman, J.H. Krolik, and S.C. Noble. Astrophys. J. 769, 156 (2013). doi:10.1088/0004-637X/769/2/156.

10. J.M. Miller, A.C. Fabian, J. Kaastra, T.R. Kallman, A.L. King, D. Proga, J. Raymond, and C.S. Reynolds. Astrophys. J. 814, 87 (2015). doi:10.1088/0004637X/814/2/87.

11. J. Deprince, S. Fritzsche, T.R. Kallman, P. Palmeri, and P. Quinet. AIP Conf. Ser. 1811, 040002 (2017). doi:10.1063/1.4975718.

12. I.P. Grant, B.J. McKenzie, P.H. Norrington, D.F. Mayers, and N.C. Pyper. Comput. Phys. Commun. 21, 207 (1980). doi:10.1016/0010-4655(80)90041-7.

13. B.J. McKenzie, I.P. Grant, and P.H. Norrington. Comput. Phys. Commun. 21, 233 (1980). doi:10.1016/0010-4655(80)90042-9.

14. I.P. Grant. Meth. Comput. Chem. 2, 1 (1988).

15. F.A. Parpia, C.F. Fischer, and I.P. Grant. Comput. Phys. Commun. 94, 249 (1996). doi:10.1016/0010-4655(95)00136-0.

16. S. Fritzsche. Comput. Phys. Commun. 183, 1523 (2012).

17. I.P. Grant. Relativistic quantum theory of atoms and molecules. Springer. 2007. doi:10.1007/978-0-387-35069-1.

18. H. Nguyen, M. Koenig, D. Benredjem, M. Caby, and G. Coulaud. Phys. Rev. A, 33, 1279 (1986). doi:10.1103/PhysRevA.33.1279.

19. J.C. Weisheit. Adv. At. Mol. Phys. 25, 101 (1989). doi:10.1016/S0065-2199(08) 60084-6.

20. T.N.Chang andT.K.Fang.Phys. Rev.A, 88, 023406(2013). doi:10.1103/PhysRevA. 88.023406.

21. M. Belkhiri, C.J. Fontes, and M. Poirier. Phys. Rev. A, 92, 032501 (2015). doi: 10.1103/PhysRevA.92.032501.

22. A. Piel. Plasma physics. An introduction to laboratory, space and fusion plasmas. Springer-Verlag. 2010. doi:10.1007/978-3-642-10491-6.

23. M. Das, B.K. Sahoo, and S. Pal. Phys. Rev. A, 93, 052513 (2016). doi:10.1103/ PhysRevA.93.052513.

24. F.Y. Khattak, O.A.M.B. Percie, du Sert, F.B. Rosmej, and D. Riley. J. Phys. Conf. Ser. 397, 012020 (2012). doi:10.1088/1742-6596/397/1/012020.

25. T.R. Kallman and M. Bautista. Astrophys. J. Suppl. Ser. 133, 221 (2001). doi:10. $1086 / 319184$.

26. M. Bautista and T.R. Kallman. Astrophys. J. Suppl. Ser. 134, 139 (2001). doi:10. $1086 / 320363$. 